\title{
Ab Initio Molecular Orbital Calculations for the Double-Stranded Helices of Poly(ethylenimine) Oligomer Models
}

\author{
Hiroshi KuSANAGI* \\ Unitika Research Laboratories Inc., Uji, Kyoto 611, Japan
}

(Received October 2, 1995)

\begin{abstract}
An attempt was made to calculate the energetical stability of the double-stranded helix of poly(ethylenimine) oligomers, a model compound for polymer double-stranded helix, by using the $a b$ initio molecular orbital method. Calculated total energy of double-stranded helix for pentamer is stabilized, $\Delta E=-1.83 \mathrm{kcal} \mathrm{mol}^{-1}$ of monomer unit, with $\mathrm{N}-\mathrm{H} \cdots \mathrm{N}$ hydrogen bonding between the twisted two pentamer chains in the double-strand, in comparison with twice of the isolated single helix. Moreover, it was found that the calculated helix radii for pentamer are about $4-10 \%$ larger than those of polymer helix determined by X-ray analysis. Tight double-stranded helix structure of polymer may originate from the long range intermolecular-interactions along helix direction.

KEY WORDS Ab Initio Calculation / Double-Stranded Helix / Poly(ethylenimine)/Oligomer / Stabilizing Energy /
\end{abstract}

$\mathrm{X}$-Ray structural analysis of poly(ethylenimine) (PEI) $-\left(\mathrm{NHCH}_{2} \mathrm{CH}_{2}\right)_{n}$ - reveals that the polymer chains exist as double-stranded helices. ${ }^{1}$ The NH groups of PEI can behave as both proton donors and proton acceptors for hydrogen bonding. Therefore, the formation of PEI double-stranded helix is considered to be stabilized with $\mathrm{N}-\mathrm{H} \cdots \mathrm{N}$ hydrogen bonding between the two polymer chains in the double-strand, as same as in DNA doublestranded helices. However, there are no studies on the energy calculation of the PEI double-stranded helix structure determined by X-ray analysis. We are aiming to evaluate the stabilizing mechanism of double-stranded helix of PEI in terms of the ab initio molecular orbital (MO) calculations. At the first stage of our purpose, in this study, $a b$ initio MO calculations were made to evaluate the energetical stability of the double-stranded helix of PEI oligomers, a model compound for polymer double-stranded helix. ${ }^{2}$

\section{METHODS}

$A b$ initio molecular orbital calculations were carried out with using the version of the Hondo- 8 system of programs. Both STO-3G and 4-31G basis sets were adopted, and ab initio SCF-MO method was used as the calculation procedure.

Two kinds of double-stranded helix models for PEI dimers $\left(\mathrm{CH}_{3} \mathrm{NHCH}_{2} \mathrm{CH}_{2} \mathrm{NHCH}_{3}: 18\right.$ atoms) and pentamers $\left(\mathrm{CH}_{3}\left(\mathrm{NHCH}_{2} \mathrm{CH}_{2}\right)_{4} \mathrm{NHCH}_{3}\right.$ : 42 atoms $)$ were constructed as model compounds for polymer, using the structural parameters determined by $\mathrm{X}$-ray analysis as shown in Figure 1. Adopting these double-stranded helix models as the initial structure, geometry optimizations of the structure, i.e., energy minimization of total energy $E$, were performed with STO basis set only for saving the computational time. The convergence threshold on the maximum gradient component was assumed to the default value 0.0005 in the program. Since the doublestranded helix of PEI has the two-fold rotation axis coinciding to the helix axis, i.e., $\mathrm{C}_{2}$-symmetry, each single helix chain constituting the double-stranded helix has the same geometry (denoted to $A$ ). For the optimized structure of double-stranded helix model, total energies $E(A \cdots A)$ were calculated with STO-3G and also 4-31G basis set with higher accuracy. On the other hand, total energy $E(A)$ of the single helix model was calculated using one half of the optimized structure. The interaction energy $\Delta E$ (=stabilizing energy) between the two molecular chains in the double-stranded helix, in other words, the complex formation energy can be calculated as follows, ${ }^{3}$

$$
\Delta E=[E(A \cdots A)-2 E(A)] / 2
$$

IBM computer work station was used for the calculation.

\section{RESULTS AND DISCUSSION}

\section{Double-Stranded Helix of PEI Dimer Model}

Optimization for the geometry of the double-stranded helix of PEI dimer model was made under the condition of keeping the $\mathrm{C}_{2}$-symmetry during the optimization process. Total energy $E$ was converged after 123 cycles. Table I lists the total energies $E(A \cdots A), E(A)$, and stabilizing energy $E$. For the double-stranded helix of PEI dimers, the stabilizing energy was calculated to be $\Delta E=-1.45 \mathrm{kcal} \mathrm{mol}^{-1}$ of single helix in terms of the 4-31G basis set with higher accuracy. This single helix of PEI dimer has two $\mathrm{N}-\mathrm{H}$ groups composed of $\mathrm{N}-$ $\mathrm{H} \cdots \mathrm{N}$ hydrogen bond and free $\mathrm{N}-\mathrm{H}$ bond types (see

Table I. Optimized total energy $(E)$, stabilizing enegy $(\Delta E)$, and hydrogen-bond energy $\left(\Delta E_{\mathrm{H}}\right)$ of double-stranded helix of PEI dimer model compound

\begin{tabular}{|c|c|c|c|c|}
\hline \multirow{2}{*}{ Basis set } & $E(A \cdots A)$ & $E(A)$ & $\Delta E^{\mathrm{a}}$ & $\Delta E_{\mathrm{H}}^{\mathrm{b}}$ \\
\hline & $\mathrm{au}$ & $\mathrm{au}$ & $\mathrm{kcal} \mathrm{mol}^{-1}$ & $\mathrm{kcal} \mathrm{mol}^{-1}$ \\
\hline STO-3G & -528.1676 & -264.0827 & -0.68 & -0.68 \\
\hline $4-31 \mathrm{G}$ & -533.8385 & -266.9169 & -1.45 & -1.45 \\
\hline
\end{tabular}

${ }^{\mathrm{a}} \Delta E=[E(A \cdots A)-2 E(A)] / 2 \cdot{ }^{\mathrm{b}} \Delta E_{\mathrm{H}}=\Delta E / n_{\mathrm{H}}$, where $n_{\mathrm{H}}$ is the number of hydrogen bonds. 

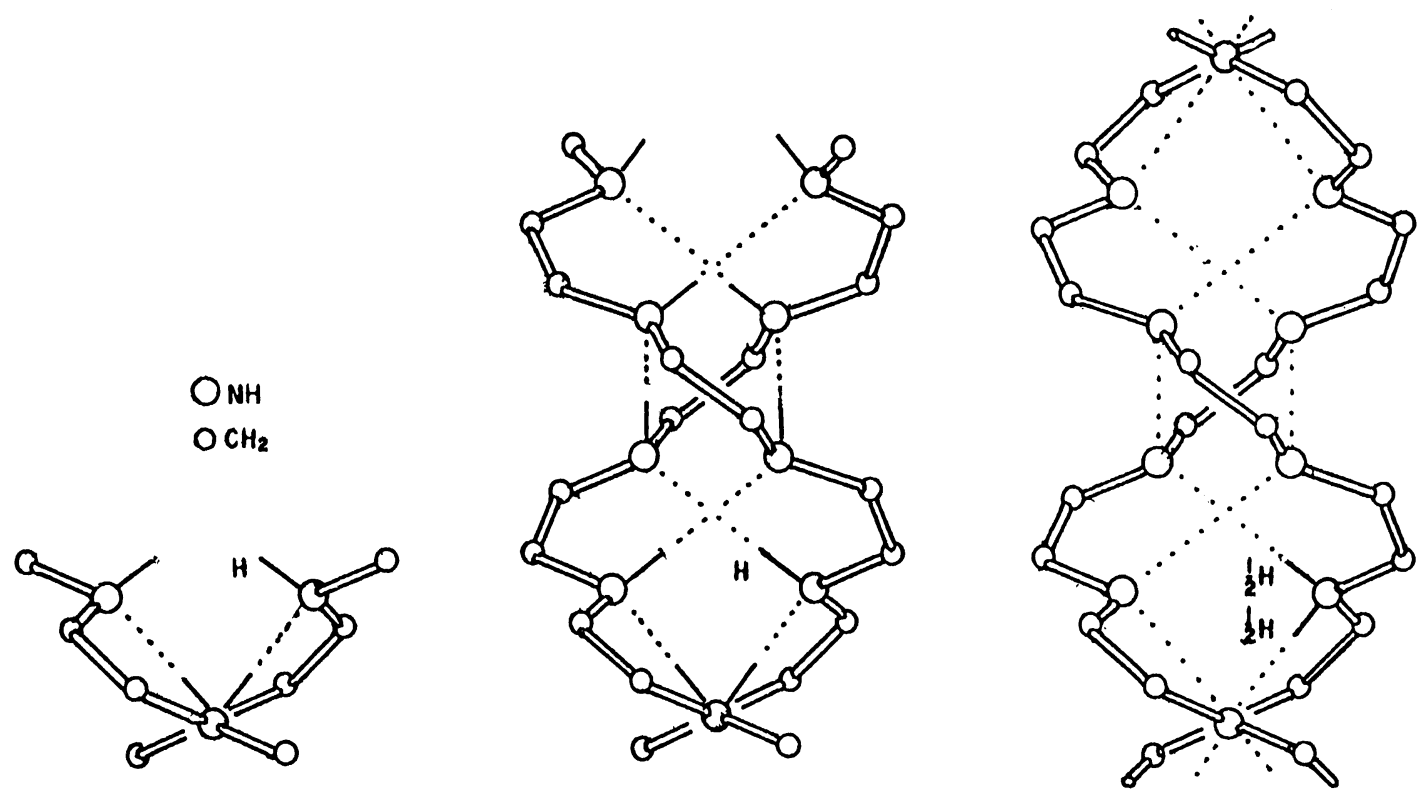

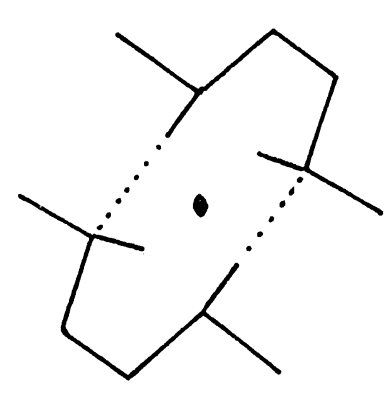

(a)

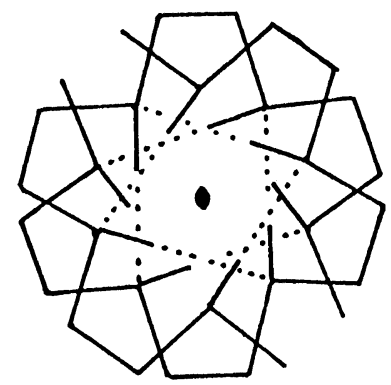

(b)

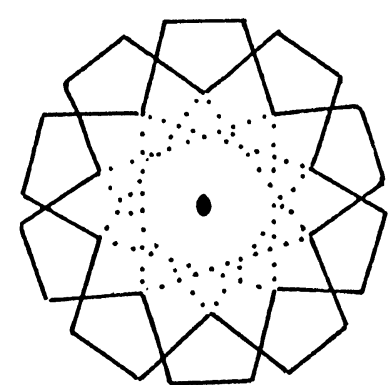

(c)

Figure 1. Dobuble-stranded helical structure models for (a) dimer, (b) pentamer, and (c) polymer of PEI.

Figure 1). Therefore, the stabilizing energy $\Delta E_{\mathbf{H}}=-1.45$ $\mathrm{kcal} \mathrm{mol}^{-1}$, divided by the number hydrogen bond, is corresponding to the $\mathrm{N}-\mathrm{H} \cdots \mathrm{N}$ hydrogen bond energy. This value is in good agreement with the standard value $\sim-1.92 \mathrm{kcal} \mathrm{mol}^{-1}$ for $\mathrm{N}-\mathrm{H} \cdots \mathrm{N}$ hydrogen bond. ${ }^{4}$ Dimer model compound is too small to form the helix. Therefore, the calculation was made using PEI pentamer model compound shown in Figure 1.

\section{Double-Stranded Helix of PEI Pentamer Model}

Optimization was carried out in the same manner for dimer model, but was stopped when the maximum gradient component had reached to 0.002 after 33 cycles, because of restriction of computational time. The change of total energy $E(A \cdots A)$ at the final optimization process was smaller than $0.00001 \mathrm{au}$. Total energies $E(A \cdots A), E(A)$, and stabilizing energies $\Delta E$ and $\Delta E_{\mathrm{H}}$ are listed in Table II. In case of pentamer model, $\Delta E_{\mathrm{H}}=-2.71 \mathrm{kcal} \mathrm{mol}^{-1}$, corresponding to hydrogen bonding energy, is considerably large, in comparison with the standard values $\sim-1.92 \mathrm{kcal} \mathrm{mol}^{-1}$ for $\mathrm{N}-\mathrm{H} \cdots \mathrm{N}$ hydrogen bond. ${ }^{4}$ For the reasons, it is considered that the long range interaction energies along the helix axis between the intertwined two chains may contribute to this stabilizing energy of pentamaer model ${ }^{5}$ On the other
Table II. Optimized total energy $(E)$, stabilizing enegy $(\Delta E)$, and hydrogen-bond energy $\left(\Delta E_{\mathrm{H}}\right)$ of double-stranded helix of PEI pentamer model compound

\begin{tabular}{|c|c|c|c|c|}
\hline \multirow{2}{*}{ Basis set } & $E(A \cdots A)$ & $E(A)$ & $\Delta E^{\mathrm{a}}$ & $\Delta E_{\mathbf{H}}{ }^{\mathrm{b}}$ \\
\hline & $\mathrm{au}$ & $\mathrm{au}$ & $\mathrm{kcal} \mathrm{mol}^{-1}$ & $\mathrm{kcal} \mathrm{mol}^{-1}$ \\
\hline STO-3G & -1316.9545 & -658.4673 & -6.24 & -1.56 \\
\hline $4-31 G$ & -1331.0809 & -665.5232 & -10.82 & -2.71 \\
\hline
\end{tabular}

${ }^{\mathrm{a}} \Delta E=[E(A \cdots A)-2 E(A)] / 2{ }^{\mathrm{b}} \Delta E_{\mathrm{H}}=\Delta E / n_{\mathrm{H}}$, where $n_{\mathrm{H}}$ is the number of hydrogen bonds.

hand, this interaction energies must be small in short helix of PEI dimers.

With respect to the long range interaction effect of polymer chains, $a b$ initio MO calculation of PEI pentamer helix gives an interesting inspection on the molecular structure of double-stranded helix of PEI. Table III lists the helix parameters, which were obtained by averaging the structure parameters of MO calculation on the pentamer model, in comparison with the observed parameters on the polymer double-stranded helix obtained by $\mathrm{X}$-ray analysis. Helix radii, $r\left(\mathrm{CH}_{2}\right)=2.777 \AA$ and $r(\mathrm{NH})=1.705 \AA$, of main chain carbon and nitrogen atoms of pentamer model are about $4-10 \%$ larger than 
Table III. Helix parameters of PEI doubel-stranded helix for pentamer and polymer

\begin{tabular}{lcc}
\hline & $\begin{array}{c}\text { Pentamer helix } \\
\text { MO calcd }^{\mathrm{a}}\end{array}$ & $\begin{array}{c}\text { Polymer helix } \\
\text { X-ray obsd }\end{array}$ \\
\hline $\begin{array}{l}\text { Helix radius } / \AA \\
r\left(\mathrm{CH}_{2}\right)\end{array}$ & 2.777 & \\
$r(\mathrm{NH})$ & 1.705 & 2.670 \\
Helical pitch & & 1.550 \\
$\quad$ of monomer $/ \AA$ & 1.977 & 1.916 \\
\hline
\end{tabular}

${ }^{a}$ Averaged values calculated for pentamer model compound. ${ }^{b}$ From ref 1 .

those of polymer helix determined by X-ray analysis. Considering the accuracy of ab initio MO calculations, ${ }^{6}$ the double stranded helix of PEI polymer is slender than these of PEI pentamer model compound. This result gives an evidence that the long range intermolecularinteractions along the helix axis direction make more close contact between the intertwisted two chains in the double-stranded helix.

Acknowledgment. This work is based in part on results obtained from the MOTECC Package.

\section{REFERENCES}

1. Y. Chatani, T. Kobatake, H. Tadokoro, and R. Tanaka, Macromolecules, 15, 170 (1982).

2. I. Ando, "Solid NMR of Polymer," Kodansha, Tokyo, 1994, p 24.

3. J. Yonezawa, T. Nagata, H. Kato, S. Imamura, and K. Morokuma, "Introduction to Quantam Chemistry," 3rd ed, Kagaku-Dojin, Tokyo, 1985, p 610.

4. Y. Sasada, Y. Otani, and Y. Saito, "Introduction to Molecular Science of Crystal," Kodansya Scientific, Tokyo, 1991, p 97.

5. J. Yonezawa, T. Nagata, H. Kato, S. Imamura, and K. Morokuma, "Introduction to Quantum Chemistry," 3rd ed, Kagaku-Dojin, Tokyo, 1985, p 568.

6. K. Ohno and K. Morokuma, "Quantum Chemistry Literature Data Base," Elsevier, Amsterdam, Holland, 1982, p 418. 